\title{
Concern about body integrity and a good physical appearance were associated with poorer psycho- social adjustment after treatment for breast cancer
}

Carver CS, Pozo-Kaderman C, Price AA, et al. Concern about aspects of body image and adjustment to early stage breast cancer. Psychosom Med 1998 Mar/Apr;60:168-74.

\section{Question}

Are concerns about aspects of body image associated with adjustment to surgery for breast cancer?

\section{Design}

Inception cohort followed up for 1 year.

\section{Setting}

University of Miami oncology clinic and a private practice in Florida, USA.

\section{Patients}

77 patients with stage I or stage II breast cancer. Eligibility criteria were English fluency, no concurrent disease, and no previous psychiatric history or previous cancer. 66 women $(86 \%$, mean age 53 y) completed 5 of 6 interviews and were included in the analyses. Of the 66 women, 34 had single modified radical mastectomies, 6 had bilateral mastectomies, and 26 had lumpectomies. 21 subsequently had radiation therapy, 15 had chemotherapy, and 34 received tamoxifen. 20 mastectomy patients had reconstructive surgery: 19 within the first 3 months, 1 after a 5 month delay.

\section{Assessment of prognostic factors}

Interviews were done before surgery, 7-10 days after surgery, and 3,6, and 12 months later. The initial interview included an assessment of demographic variables and a measure of investment in body image (trait-like concern about physical appearance and about body integrity). Investment in body image was reassessed at 12 months. All interviews other than the first one included a measure of emotional adjustment. The initial, and 3, 6, and 12 month follow up interviews included a measure of psychosexual adjustment. The follow up interviews also included a measure of the extent to which the treatment for the illness was perceived to be interfering with social and recreational activities.

\section{Main outcome measures}

Psychosocial adjustment including measures of investment in body image, emotional and psychosexual adjustment, disruption of activities, and alienation from self.

\section{Main results}

All analyses were controlled for age. Concern about appearance was consistently related to distress at a low to moderate level at 3 (standardised regression coefficient $0.27, \mathrm{p}<0.05$ ) and 6 months (coefficient $0.30, \mathrm{p}<0.05$ ) follow up. Women who were high in concern about appearance expressed a consistent and stable sense of distress throughout the follow up period. Investment in appearance also made women more resilient against deterioration in their perceptions of attractiveness (coefficient at 12 month follow up $0.30, \mathrm{p}<0.05$ ). Concern about body integrity was not related to emotional distress at any measurement time except for the initial interview, but it was related to adverse effects on social and recreational activities (coefficient at 12 months follow up 0.36, $<<0.02$ ), to deterioration in feelings of sexual desirability, and to feelings of alienation from the self (coefficient at 6 months follow up 0.40, p $<0.01$ ).

\section{Conclusions}

Women whose sense of self was highly invested in their physical appearance before surgery for breast cancer reported more distress during the subsequent year but less disruption of their sense of femininity and sexual desirability. Women who reported being invested in the sense of body integrity reported no greater emotional distress but had more disruption in social activities and a stronger sense of not being themselves.

Source of funding: American Cancer Society.

For correspondence: Dr CS Carver, Department of Psychology, PO Box 248185, University of Miami, Coral Gables, FL 33124-2070, USA. Fax +1 3052843402.

\section{Commentary}

Treatments for cancer can be permanently or temporarily disfiguring and various dimensions of self (eg, self esteem, self confidence, sense of self worth, body image) may be acutely threatened by both the disease and its treatment. This clinical experience has not always been reflected in research: an omission which Carver et al begin to address in this study. Although the results raise more questions than answers, the authors are to be congratulated on moving away from yet another survey of distress towards attempting to understand some of the mechanisms that might underlie such distress.

As treatment for breast cancer varies in the degree of damage to a physically and socially highly visible and valued part of the body, the authors set out to test the hypothesis that women whose sense of self is highly invested in their physical appear- ance may be more at risk for post-surgical emotional distress than women with a lower investment. They make an important distinction between body image (ie, an individual's perception of what her body is like) and concern over, or investment in, body image (ie, how much body image actually matters to the person). They devised a new measure (the Measure of Body Apperception-MBA) which has 2 dimensions of concerns-physical appearance and a sense of body integrity. Carver et al acknowledge the preliminary nature of the data arising from this measure, and it would be prudent to avoid drawing too many definite conclusions from the data presented.

However, the suggestion that concerns about appearance may have a buffering effect on femininity and sexual desirability demands further investigation, as does the link with alienation from the self. Likewise, the suggestion that women who reported a greater sense of investment in the sense of body integrity preoperatively experienced a greater sense of "not being themselves" is intriguing. Although developed in the context of breast cancer, there seems to be no good reason why the MBA should not find application to other cancers. Carver et al have opened up a necessary debate about the concept of body image and cancer. They have also confirmed the importance for all clinicians of the necessity for taking the patient's self image seriously-and not dismissing such concerns as mere vanity.

$$
\begin{array}{r}
\text { Peter Harvey, PhD } \\
\text { Birmingham Oncology Centre } \\
\text { Queen Elizabeth Hospital } \\
\text { Birmingham, UK }
\end{array}
$$

